\title{
Waffle Method for optimizing cryo-FIB-milling
}

Kotaro Kelley ${ }^{1}$, Pattana Jaroenlak ${ }^{2}$, Ashleigh Raczkowski ${ }^{3}$, Edward Eng ${ }^{4}$, Gira Bhabha ${ }^{5}$, Clint Potter ${ }^{4}$, Bridget Carragher ${ }^{4}$ and Alex Noble ${ }^{4}$

${ }^{1}$ New York Structural Biology Center, United States, ${ }^{2}$ NYU, United States, ${ }^{3}$ NYSBC, New York, New York, United States, ${ }^{4}$ New York Structural Biology Center, New York, New York, United States, ${ }^{5}$ New York University School of Medicine, United States

Cryo-electron tomography (cryo-ET) allows researchers to directly observe proteins and subcellular structures in their native 3D cellular contexts at nanometer and sub-nanometer resolution. This technology can reveal cellular phenotypes and complete inner workings of cells in natural and perturbed conditions. The highest resolution 3D in situ structural method is to prepare cells by cryo-focused ion beam milling/scanning electron microscope (cryo-FIB/SEM) (Figure 1a-c) followed by cryo-ET collection. Cryo-FIB/SEM sample preparation entails identifying regions of interest by 3D (cryo-)light microscopy (LM) within the bulk sample, slicing out thin cross-sections called a lamellae, and performing cryo-ET. Individual objects of interest are directly observable in tomograms and repeated objects can be computationally solved at higher resolution. However, two major issues prevent visual proteomics of cell interiors by cryo-ET from becoming routinely accessible. First, sample preparation and data collection are low throughput and error prone. Second, object identification in crowded 3D cellular interiors remains a critical bottleneck.

To increase sample preparation and collection throughput, we recently developed the Waffle Method, which allows large numbers of small suspension cells to be simultaneously prepared by cryo-FIB/SEM and collected by cryo-ET at nanometer-resolution in 3D (Figure 1d-i). The Waffle Method produces lamellae tens of microns across (Figure 1f), compared to only a few microns for conventionally-prepared lamellae (Figure 1b,c), an order of magnitude throughput increase. To accomplish this, sample is applied in bulk to the cryo-EM grid and high-pressure frozen before a layer of platinum is applied and the grid is milled. First, trenches perpendicular to the grid are milled into the large slab of sample in each square of interest, leaving a 30 micron chink of sample between each set of trenches (Figure 1e). The grid is then tilted for shallow-angle milling and milled carefully with the leading edge of the FIB-beam always contacting the platinum first to minimize curtaining. Partway through milling, a specially-designed notch is cut through one side of the lamella to allow for grid flexibility and lamella stability, which substantially increases lamellae survivability. Finally, lamella fine milling and polishing is performed. Lamellae may be milled in parallel to avoid cross-contamination.

We find that the Waffle Method generally solves the following problems compared to conventional cryoFIB/SEM milling: any issues with specimen preferred orientation are resolved, low milling throughput due to specimen size or concentration are resolved, and specimen vitrification issues are resolved. We envision that the Waffle Method may become a way of performing cryo-liftout on the grid, particularly for tissue specimens, due to the reduced technical and equipment requirements, and that it may become automated due to all operations being performed on a single grid. 


\section{Conventional cryo-FIB/SEM}

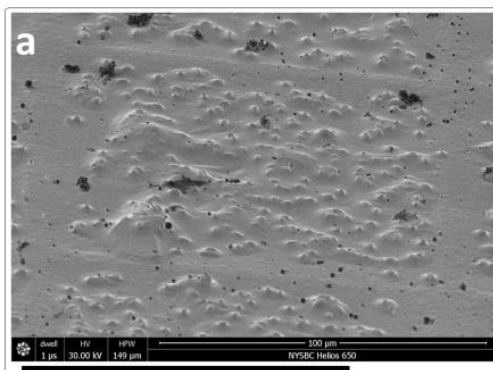

$100 \mu \mathrm{m}$

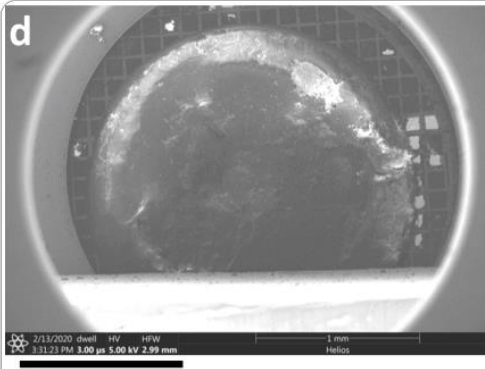

$1 \mathrm{~mm}$

g

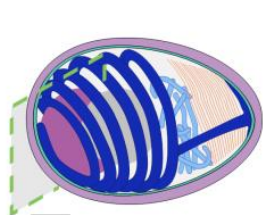

$\overline{500} \mathrm{~nm}$

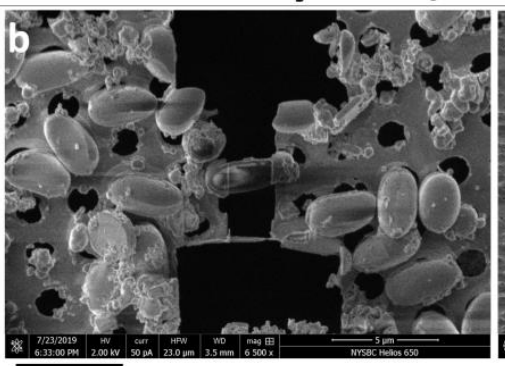

$5 \mu \mathrm{m}$

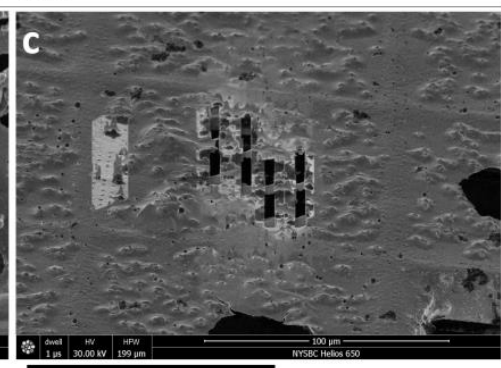

$100 \mu \mathrm{m}$

\section{Waffle method}

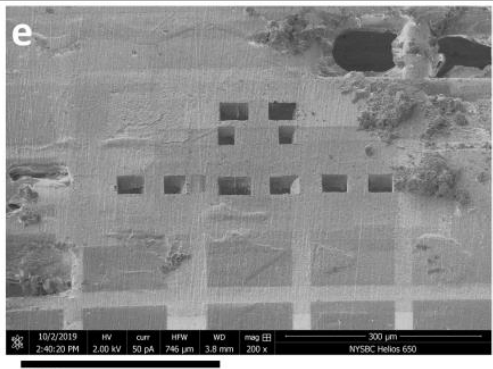

h



$20 \mu \mathrm{m}$
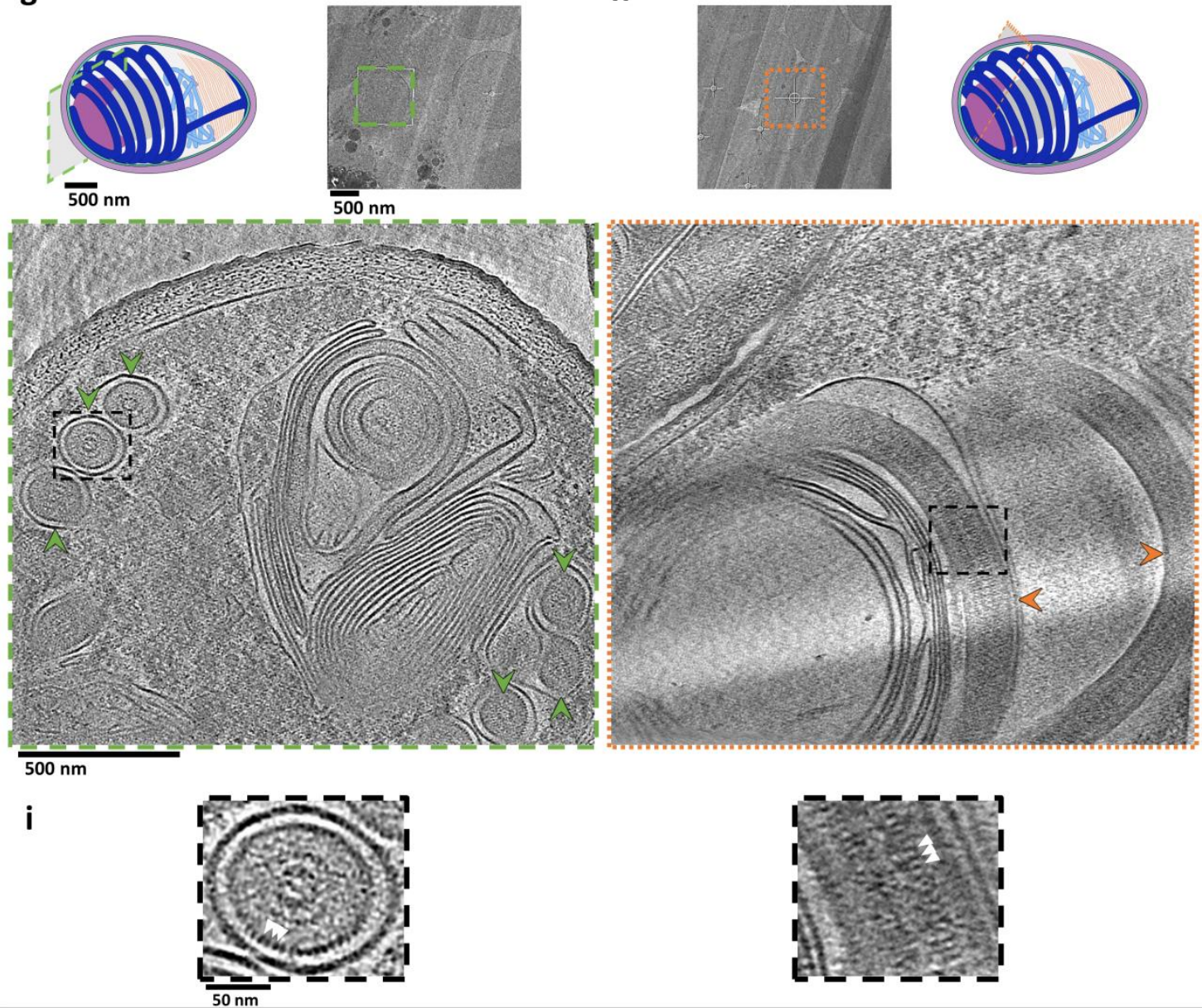
Figure 1. Example showing how the Waffle Method applied to small suspension cells significantly improves cryoFIB/SEM throughput and solves preferred orientation issues. (a-c) Small cells milled by conventional cryoFIB/SEM. The cells are individually-milled due to low concentration, leading to low throughput and only one orientation. (a) FIB image of cells in a cryo-EM grid square. (b) SEM image of an individually-milled cell $(\sim 1.5 \mathrm{x}$ $3 \mu \mathrm{m}$ ). (c) FIB image of several individually-milled cells. (d-i) Small cells prepared using the Waffle Method. (d) SEM image of a Waffle Method grid at low-magnification showing the whole grid. (e) SEM image of a Waffle Method grid with several trenches prepared. (f) SEM image of a completed Waffle Method lamella $(\sim 30$ x $20 \mu \mathrm{m})$ showing dozens of cell cross-sections. (g) A low-magnification TEM image of a Waffle Method lamella alongside a schematic diagram of a spore with the high-magnification cryo-ET collection area approximated (green outlined cross-section). Below is a slice-through of the high-magnification, denoised tomogram with arrows showing axial views of the polar tube in the spore (green arrows). The polar tube in the schematic diagram is colored dark blue. (h) A low-magnification TEM image of a Waffle Method lamella alongside a schematic diagram of a spore with the high-magnification cryo-ET collection area approximated (orange outlined cross-section). The spore crosssection is roughly orthogonal to the spore in $(\mathrm{g})$, as the diagrams show. Below is a slice-through of the highmagnification, denoised tomogram with arrows showing side views of the polar tube in the spore (orange arrows). (i) The dotted black line insets in (e) and (f) magnified by $4 \mathrm{x}$ highlighting the $\sim 2.5 \mathrm{~nm}$ features on the second cylindrical layer of the polar tubes (white arrows).

\section{References}

Arnold, J. et al. Site-Specific Cryo-focused Ion Beam Sample Preparation Guided by 3D Correlative Microscopy. Biophys. J. 110, 860-869 (2016).

Bepler, T. et al. Topaz-Denoise: general deep denoising models for cryoEM and cryoET. Nat. Commun. 11, 5208 (2020).

Burt, A. et al. Tools enabling flexible approaches to high-resolution subtomogram averaging. bioRxiv 2021.01.31.428990 (2021).

Chen, M. et al. A complete data processing workflow for cryo-ET and subtomogram averaging. Nat. Methods 16, 1161-1168 (2019).

Himes, B. A. \& Zhang, P. emClarity: software for high-resolution cryo-electron tomography and subtomogram averaging. Nat. Methods 15, 955-961 (2018).

Kelley, K. et al. Waffle method: A general and flexible approach for FIB-milling small and anisotropically oriented samples. bioRxiv 2020.10.28.359372 (2020).

Marko, M. et al. Focused-ion-beam thinning of frozen-hydrated biological specimens for cryo-electron microscopy. Nat. Methods 4, 215-217 (2007).

Noble, A. J. \& Stagg, S. M. Automated batch fiducial-less tilt-series alignment in Appion using Protomo. J. Struct. Biol. 192, 270-278 (2015).

Rigort, A. et al. Focused ion beam micromachining of eukaryotic cells for cryoelectron tomography. Proc. Natl. Acad. Sci. 109, 4449-4454 (2012).

Schaffer, M. et al. A cryo-FIB lift-out technique enables molecular-resolution cryo-ET within native Caenorhabditis elegans tissue. Nat. Methods 16, 757-762 (2019).

Tacke, S. et al. A streamlined workflow for automated cryo focused ion beam milling. bioRxiv 2020.02.24.963033 (2020).

Tegunov, D. et al. Multi-particle cryo-EM refinement with M visualizes ribosome-antibiotic complex at $3.5 \AA$ in cells. Nat. Methods 1-8 (2021).

Turoňová, B. et al. Efficient 3D-CTF correction for cryo-electron tomography using NovaCTF improves subtomogram averaging resolution to 3.4 Å. J. Struct. Biol. (2017). 